\title{
A process mining approach to analyse user behaviour
}

\author{
Laura Măruşter and Niels Faber \\ Faculty of Management and Organization, University of Groningen \\ PO Box 800, 9700 AV Groningen, The Netherlands
}

\begin{abstract}
Designing and personalising systems for specific user groups encompasses a lot of effort with respect to analysing and understanding user behaviour. The goal of our paper is to provide insights about patterns of behaviour of specific user groups; we consider farmers as a specific user group, during the usage of a decision support system supporting cultivar selection - OPTIRas ${ }^{\mathrm{TM}}$. We analyse users' patterns of behaviour by combining these insights with decision making theories, and previous work concerning the development of farmer groups. We provide a method of automatically analysing the logs resulted from the usage of the decision support system by means of process mining. The results of our analysis can be used to support the redesigning and personalization of decision support systems in order to address specific users, namely farmer's characteristics.
\end{abstract}

Keywords: modelling user behaviour, decision support systems, process mining, farmer groups, personalization

\section{Introduction}

As the on-line services and Web-based information systems proliferate in all domains of activities, it became increasingly important to model user behaviour and personalising, so that these systems will appropriately address user characteristics.

In this sense, particular topics are addressed by research in humancomputer interaction (HCI) and user-system interaction (USI), such as the discovering of user behaviour or navigation styles $\mathrm{s}$ (Herder \& Juvina, 2005; Juvina \& Herder, 2005a; Menasalvas et al., 2003; B. Balajinah and S.V. Raghavan, 2001), developing metrics involved in modelling and assessing web navigation (Juvina \& Herder, 2005b; Herder, 2002; Pauline A. Smith, 1996; John E. McEneany, 2001; Myra Spiliopoulou and Carsten Pohle, 2001), cognitive models for improving the redesign of information systems (Juvina, Oostendorp, Karbor, \& Pauw, 2005; Juvina \& Oostendorp, 2005; M. Rauterberg, 1993; M. Rauterberg and S. Schluep and M. Fjeld, 1998; Bollini, n.d.; Neil A. Ernst and Margaret-Anne Story and Polly Allen, 2005; Manfred Thüring and Jörg Hannemann and Jörg M. Haake, 1995; Kun Chang Lee and Sangjae Lee, 2003).

Various methods have been developed to model web navigation in case of generic users. However, it became increasingly important to

(c) 2007 Kluwer Academic Publishers. Printed in the Netherlands. 
address specific user groups; by investigating the navigational patterns of these groups, the (re)design of the systems used by specific user groups can be made more effectively. In this paper, we consider farmers as a specific user group, and we analyse the patterns of behaviour to support the redesign of a decision support system.

Different projects have been initiated to support farmers to pursue their decision making activities with the aid of Information Systems (see for instance (Farm Information Systems, 2000; S. Fountas and D. Wulfsohn and B.S. Blackmore and H.L. Jacobsen and S.M. Pederson, 2006)). Although decision support systems seems to be a good solution, it is arguable whether they are fulfilling the expectations. Kuhlmann and Brodersen (Kuhlmann \& Brodersen, 2001) express their pessimism about the fast diffusion of complex information technology tools and decision support systems (DSS) among farmers; however, they suggest that multi-disciplinary work is required and DSS may still help farmers if they would be properly designed. The same discouraging voice is articulated by Hayman (Peter Hayman, 2003), who suggest that farmers' DSS usage for routine decision making is disappointing.

Researchers tried to find the factors that hamper farmers to adopt DSSs to a larger extent ((Kerr, 2004), (Berry, 1994), (Hart \& Wyatt, 1990), (Urquhart \& Rowley, 1999). For instance, Kuhlman and Brodersen (Kuhlmann \& Brodersen, 2001) found factors influencing the farmers's IT adoption, such as transaction costs, profitability of IT, user-friendly design, time requirements for IT usage, credibility, adaptation of IT to farm situations, up-to-date information, knowledge of the user. Some of their findings report that "farmers end to rely on their own experience and the advice of experts they trust", transparent input-output relation, and farmer involvement in the development process.

The goal of this work is to illustrate a methodology of analysing user behaviour using process mining (Aalst \& Weijters, 2004a). Namely, we consider farmers as a specific user group and accordingly, we aim to provide more insights about farmers' patterns of behavior during the use of decision support systems. We aim to investigate the logs resulted from using a decision support system called OPTIRas ${ }^{\mathrm{TM}}$, that aids farmers in their cultivar selection activities (AVEBE, n.d.), and to get insights into farmers' navigational behavior. We analyse farmers' patterns of behavior by combining these insights with decision making theories, and previous work concerning the development of farmer groups (N. Faber, Jorna, Haren, \& Maruster, 2006; N. R. Faber, 2006). The results of our analysis can be used to support the redesigning of DSS's in order to address specific farmer's characteristics. 
Processing logs resulting from the usage of information systems to characterize users is a common method in user-system interaction domain (Rauterberg, 2000). Focusing on farmers as a specific IT user group, it seems to become an important research issue (I. Thysen, 2000). For instance, Jensen (Allan Leck Jensen, 2001) analyzed the usage of a web-based information system for variety selection in field crops. He compared four user groups by constructing measures based on logged information. This analysis reveal interesting similarities and differences in behaviour concerning the four groups. However, no insights could be given about the most typical sequence of behaviour/navigation patterns, such that it could support the redesign of the system.

The organization of our paper is as following. In Section 2 we provide an introduction about decision making, decision support system OPTIRas $^{\mathrm{TM}}$ and related aspects to farmers, such as the development of farmers' groups, and data collection issues. In Section 3 we determine farmers' patterns of behavior with process mining techniques. The conclusions and implications for redesign are ending this paper in Section 4.

\section{Farmers and decision making}

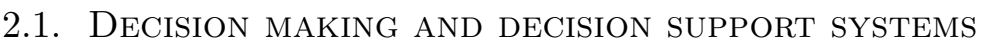

A well-known decision making model originates from Simon (Simon, 1977). He explained the human decision process using the three phases of intelligence, design, and choice. In the intelligence phase, an individual explores the issue about which he is making a decision, and determines relevant issues. The individual constructs a mental model (a representation of the problem in the mind) of the perceived issue, generally referred to as the problem space. In this problem space, the individual represents both the issue on which he is deciding and the outcome of the decision. The problem space is constructed through the breaking up of the entire decision into smaller pieces, i.e. sub-decisions. Subsequently, the individual formulates one or more possible solutions to the recognised (sub-)decisions in the design phase. Eventually, a total solution is formed in the choice phase. In this final phase of the decision process, the partial solutions are evaluated against the criteria of the outcome of the decision that need to be met. Partial solutions that best meet these criteria are selected. The selected partial solutions are combined into the complete decision. Because information that is used in the three phases is not always complete, the phases do not linearly follow upon each other. Commonly, the decision process 
is characterised by many iterations in which additional information about the issue is collected (intelligence) or more solution possibilities are explored (design). Additionally, the phases themselves are decision processes, each in itself consisting of intelligence, design, and choice phases. Mintzberg refer to a similar trichotomy: identification, development, and selection (H. Mintsberg and D. Raisingham and A. Thorêt, 1976). Specific decision making models has been developed for different kind of users. For instance, in case of farmers, decision making models have been developed by (Johnson, Halter, Jensen, \& Thomas, 1961), (Bo Öhlmér and Kent Olson and Berndt Brehmer, 1998), (S. Fountas and D. Wulfsohn and B.S. Blackmore and H.L. Jacobsen and S.M. Pederson, 2006).

Whenever these models are linear (sequential) or iterative, they all show a common structure: first, information are collected about the problem at hand, second, different candidate solutions are formed, and finally, a choice is made. No doubt that a decision support system have to address in a way or another these basic phases of the decision making process. In the following, we investigate in which way the decision support system OPTIRas ${ }^{\mathrm{TM}}$ has addressed these phases.

\subsection{OPTIRAS ${ }^{\mathrm{TM}}$}

A decision support system (DSS) is perceived as a computer system that aids people in making a decision regarding a specific domain (Klein \& Methlie, 1995). This aid is provided by the DSS through connecting to the human decision process (Turban \& Aronson, 2001).

OPTIRas $^{\text {TM }}$ system was designed to target low-yielding farmers, of the AVEBE farmer population, attempting to realize an increase in the yields of these farmers. The OPTIRas ${ }^{\mathrm{TM}}$ decision support system aids farmers in their cultivar selection, which is used in the upcoming cropping season. The selection of cultivars is one of the decisions a farmer needs to make regarding his starch potato growth. OPTIRas ${ }^{\mathrm{TM}}$ is a decision support system for cultivar selection that supports a farmer in selecting cultivars relative to cultivar characteristics. The system is developed to help low-yielding farmers. A variety of properties, relating to yield, resistance against pests and diseases, and storage, characterize a potato cultivar. The farmer's personal preferences, diseases present in the field, and cultivar properties determine his choice. OPTIRas ${ }^{\mathrm{TM}}$ assists the farmer in choosing the cultivar properties and sorting the cultivars based upon priorities. Despite its long history and body of available knowledge, potato cyst nematodes (PCN) still cause an average 150 per hectare per year of costs, in the starch potato area (about 10-15\% of farmers' net-income). Reducing the infestation to 
economic acceptable levels requires the right combination of cultivar, growth frequency, field choice, and nematicide usage. Cultivars differ greatly regarding resistance (the ability to reduce the infestation), and tolerance (the ability to resist the infestation), which are uncorrelated. A non-resistant and highly tolerant cultivar can multiply the existing PCN population by 30 , while a highly resistant and intolerant cultivar reduces the PCN population by two to three, whereby a large portion of the yield is lost. OPTIRas ${ }^{\mathrm{TM}}$ combines the PCN population level of the field and the available information of the cultivars with population dynamics of the potato cyst nematode, whereby the use of pesticides is also considered. Thus, the goal of the decision support system is to ensure that the farmer gains insight into PCN damage levels and the financial consequences of an (un)justly selected cultivar and the (un)just application of pesticides. In Figure 1 is shown a screenshot of the OPTIRas ${ }^{\mathrm{TM}}$ DSS.

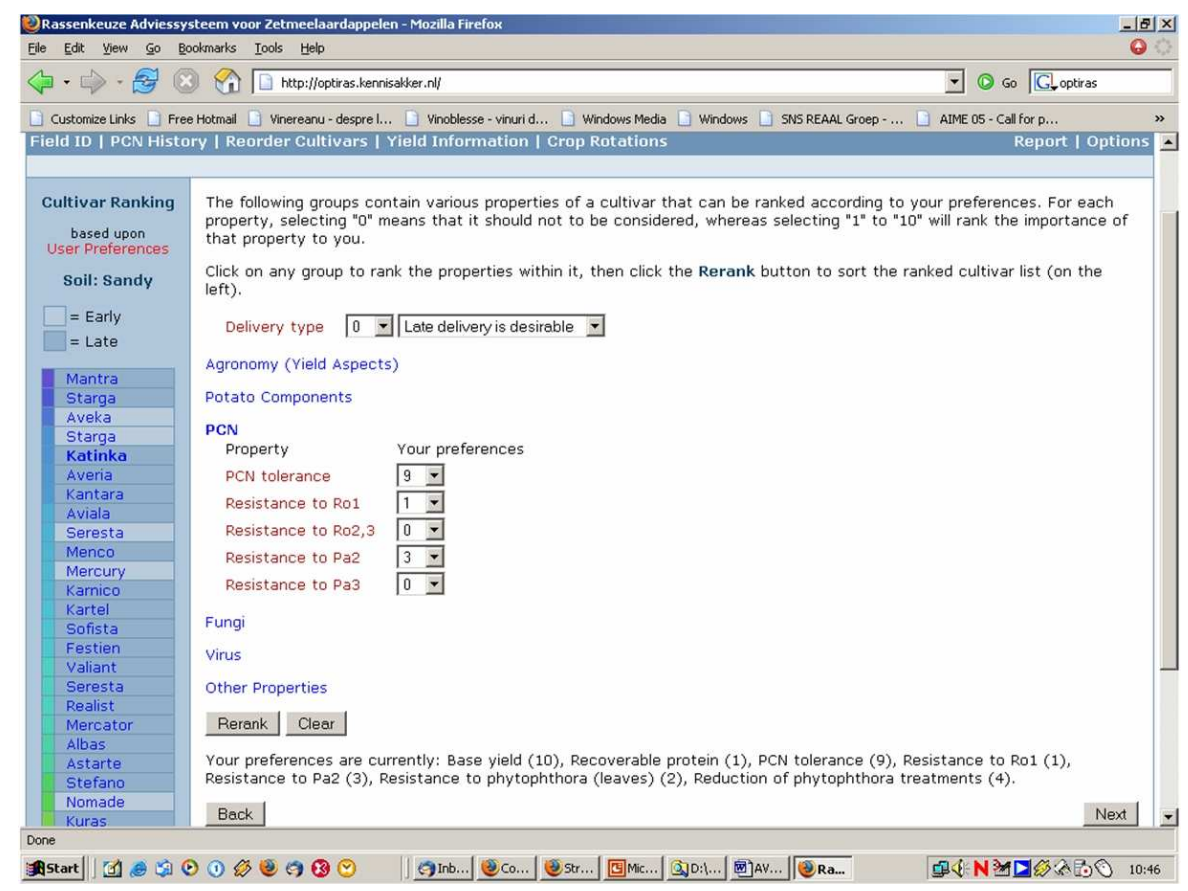

Figure 1. OPTIRas ${ }^{\mathrm{TM}}$ screenshot.

The interface of OPTIRas ${ }^{\mathrm{TM}}$ DSS consists on seven main pages: Field ID, PCN History, Reorder Cultivars, Yield Information, Crop Rotation, Report and Option. Moreover, there are also pages offering Help, Details about a specific cultivar, but they are not relevant for our analysis. 
Table I. Categorization of OPTIRas ${ }^{\mathrm{TM}}$ pages.

\begin{tabular}{|l|l|l|}
\hline 'Action' pages & 'Target' pages & Other pages \\
\hline Field ID & Yield Information & Details (Cultivar) \\
PCN History & Crop Rotations & Options \\
Reorder Cultivars & Report & Help \\
& & Logon/Logoff \\
& & Help \\
& & Save \\
\hline
\end{tabular}

Web pages do not have the same function, and this depends on the purpose of the web site. In our case, the site's goal is to provide the farmer with information about different yield scenarios, given the chosen factors. Therefore, the following types of pages can be distinguished: (i) the pages reflecting the site's goal, e.g. containing information about the yield, (ii) the pages that can lead to fulfil the site's goal, and (iii) the other pages.

In literature, this categorization is called concept hierarchies, or service-based hierarchies (Myra Spiliopoulou and Carsten Pohle, 2001). Concept hierarchies are used in market-basket analysis or to study the segmentation of companies clients. Spiliopoulou and Pohle developed in (Myra Spiliopoulou and Carsten Pohle, 2001) a service-based concept hierarchy to determine the success of a web site, which distinguishes between action pages and target pages. According to their definition, "an action page is a page whose invocation indicates that the user is pursuing the site's goal. A target page is a page whose invocation indicates that the user has achieved the site's goal".

As we stated in the previous section, the system should support the user in all phases of the decision making process. For instance, in terms of Simon decision making phases, 'Action' pages would correspond to the Intelligence phase, where an individual explores the issues about which he is making a decision, while 'Target' pages would correspond to the Design phase, where candidate solutions are developed.

We categorize OPTIRas ${ }^{\mathrm{TM}}$ pages as presented in Table I. In the following, we consider that whenever a user has visited one of the target pages (Yield Information, Crop Rotations, Report), the user has reached the goal of the web site. In Figure 2 is shown a 'Target' page of OPTIRas ${ }^{\mathrm{TM}}$, the Yield Information page.

Based on Simon's decision making phase, we proceed with the following mapping: 


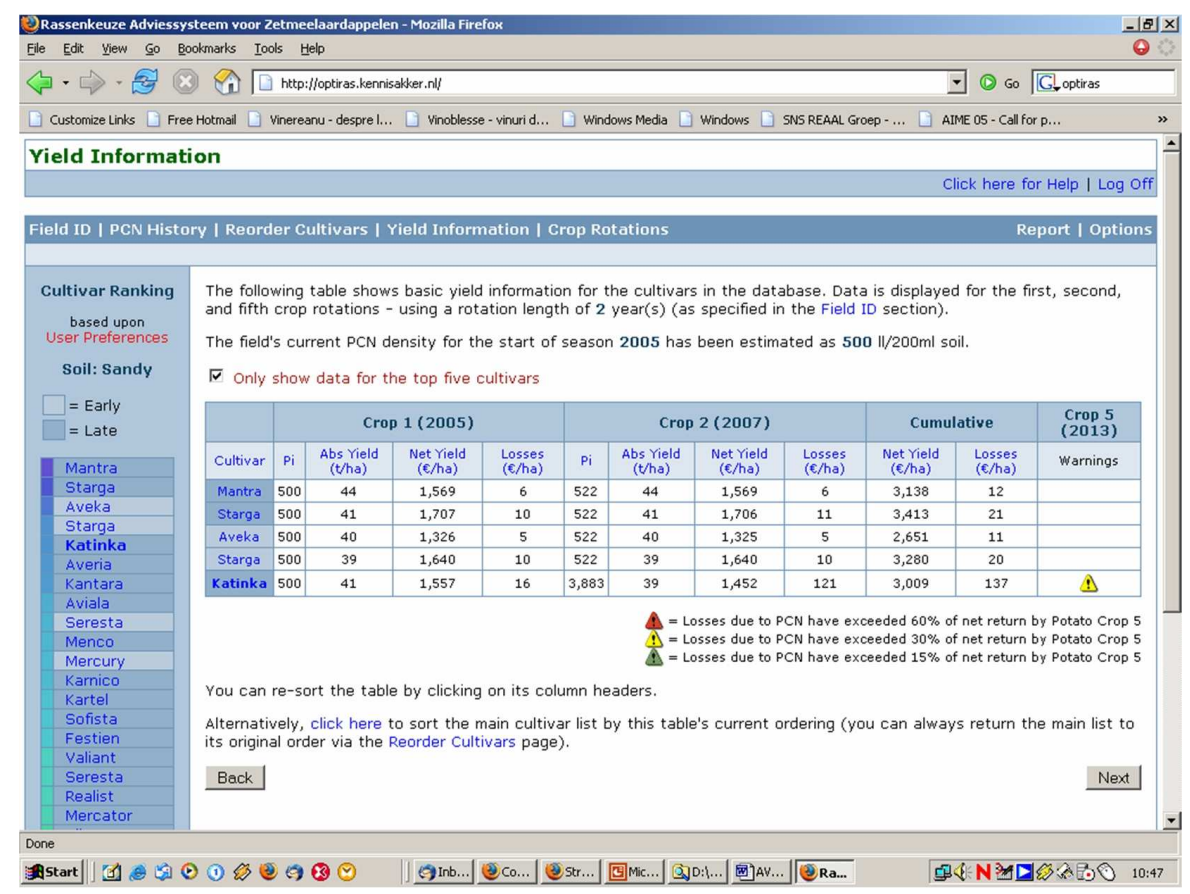

Figure 2. OPTIRas ${ }^{\mathrm{TM}}$ targetpage.

1. 'Action' pages corresponds to Intelligence phase: the farmer is supported to explore the issue (the selection of the cultivar), by specifying Field characteristics and the PCN history

2. 'Target' pages corresponds to the Design phase: the system is providing the farmer with one or more possible solutions

3. the Choice phase does not have any correspondence in the DSS; the final decision is actually taken by the farmer outside of the system

Assessing whether the DSS is "really" supporting the targeted users, or in other words, whether the site goal is fulfilled, we expect that both decision making phases, Intelligence and Design, should be performed, to the same extend. This translates into visitations of both 'Action' and 'Target' pages, to a comparable extend. In Section 3 we will investigate how the navigational patterns look like, e.g. how 'Action' and 'Target' types of pages are visited.

Analyzing users as a homogeneous group is not the most suitable basis for re-designing decision support systems that target end users, who are actually heterogeneous in nature. Therefore, when analyzing navigational patterns we take into account the fact that (i) users may belong to different target groups, and (ii) users can be split in different 
user groups. First, OPTIRas ${ }^{\mathrm{TM}}$ system was designed to be used by farmers, that can be split in three target groups, e.g. growers, extension workers, and scientists. Extension workers are people who help growers regarding all aspects of crop growth. Scientists are also using OPTIRas $^{\mathrm{TM}}$, but rather for experimental purposes. Second, farmers can be split in different farmer groups or farmer clusters, as we outline in the next section.

\subsection{FARMER GROUPS}

Approximately 2,100 Dutch starch potato farms of the Dutch AVEBE company started the 2004 growing season, all different in size and productivity. Different types of farms and farmers are assumed to exist within this population. The underlying rationale to assume different types of farmers exist is that farmers focus on different aspects of their yield, depending on their farming skills. We expected to find farmers who produce either above or below average concerning their yields in ton per hectare, and above or below average potato quality, resulting in four different groups of farmers.

Based on a cluster analysis, these four different types of farmers groups have been distinguished. The found clusters have been labelled respectively normal farmers, quality farmers, quantity farmers, and top farmers. Table II shows the differences between clusters regarding yield in tons per hectare (base weight) and potato quality in premium points. Figure 3 displays the same clusters graphically. The size of the circles refers to the contribution of the respective clusters to the total production of starch (also indicated with the mentioned percentages).

Types of farmers differ significantly concerning their farm area $(F(3,603)=5.4, p=.001)$, and area used for starch potatoes $(F(3,603)=6.7, p=.000)$. Quantity growers participate more in sugar beet growth (Pearson $\left.\chi^{2}(3, N=610)=19.6, p=.000\right)$, whereas other farmers report to grow other types of crops more

(Pearson $\left.\chi^{2}(3, N=610)=13.4, p=.004\right)$. Types of farmers also differ regarding their age $(F(3,597)=2.908, p=.034)$. Lastly, top farmers more often receive extra education $\left(\right.$ Pearson $\chi^{2}(3, N=586)=$ $33.58, p=.000)$, and more often collect information regarding starch potato growth (Pearson $\left.\chi^{2}(3, N=588)=13.86, p=.003\right)$. In relation to the sketched problems, top farmers are considered the only group able to sustain without additional measures.

Quantity and quality of a farmer's yield are the used dimensions for cluster analysis, both averaged over the last three years. The quantity of a farmer's yield is measured in tons per hectare and corrected for its starch content (base weight). The quality of a farmer's yield equals 
Table II. Clusters of farmers

\begin{tabular}{lccccc}
\hline Cluster & $N$ & \multicolumn{2}{c}{ Base weight (ton / ha) } & \multicolumn{2}{c}{ Premium points } \\
& & $\mu$ & $\sigma$ & $\mu$ & $\sigma$ \\
\hline Top farmers & 512 & 54.78 & 4.46 & 87.18 & 3.30 \\
Quantity farmers & 585 & 45.41 & 3.21 & 82.05 & 2.88 \\
Quality farmers & 391 & 41.26 & 3.65 & 88.80 & 3.05 \\
Normal farmers & 412 & 32.84 & 4.44 & 80.99 & 4.30 \\
\hline
\end{tabular}

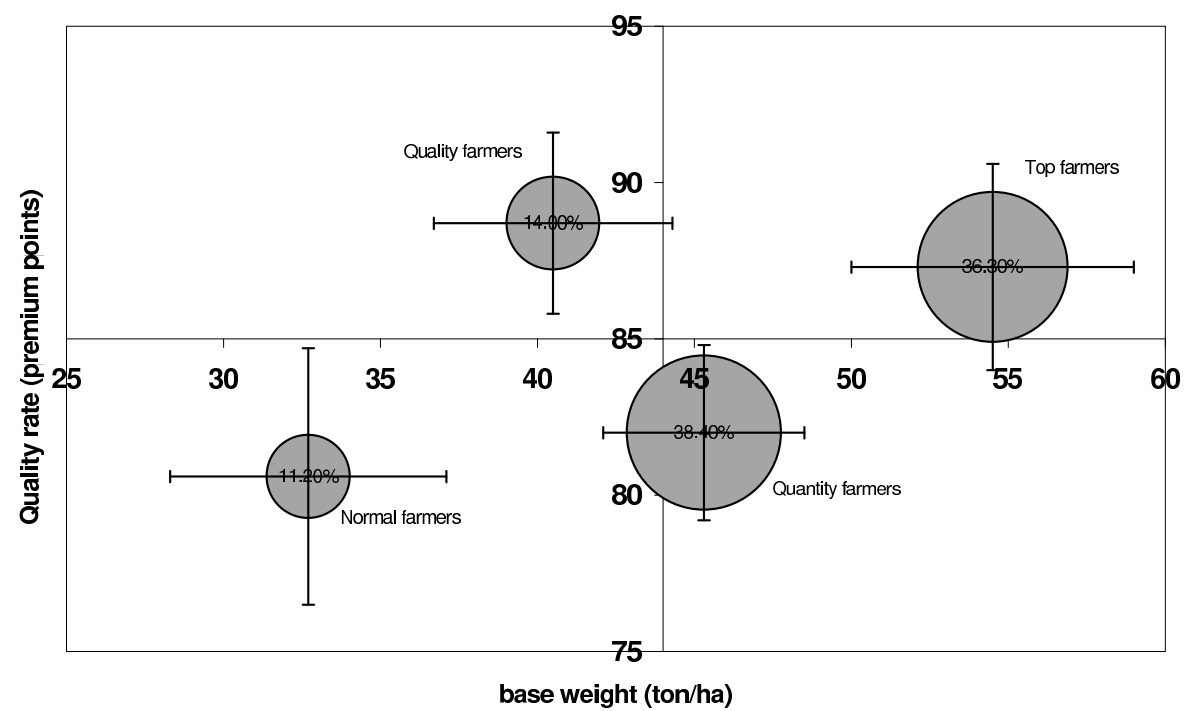

Figure 3. Farmer clusters

the premium points ascribed to the yield. These premium points are a quality measurement of potatoes a farmer delivers at factory gates. A sample of a farmer's potato delivery is valued regarding ten different dimensions, each graded between zero and ten. These include contamination (tare weight), amount of rot, heating and frost damage, and diseases. Additionally, defects due to dirt enclosure are judged. These defects include damage, growth cracks, the presence of diseases, and rust. A farmer's premium points equal the sum of the ten grades. Subsequently, the premium points are used to determine the percentage of the premium the farmer will receive as bonus, on top of the weight-based fee of his yield.

In our cluster analysis, we used multiple clustering methods ${ }^{1}$, to determine which clusters could be recognised based on farmers' yields 
and premium points. The different clustering methods were all initialised to find two to six clusters in the complete dataset of yields and premium points of the entire population of Dutch AVEBE farmers. The different methods converge at four clusters in the set. The clustering is insensitive to location and soil type.

Moreover, farmer learning styles and personalities differ between the four farmer clusters (Pieters, 2005). Farmer clusters differ significantly on the motivation scales extrinsic value and self-efficacy and on the skills scales peer learning and help seeking. Top farmers appear to have better learning attitudes relative to other farmer clusters. Quality oriented farmers seem to stay behind with respect to their learning skills, especially on the scales of peer learning, and help seeking. These farmers might perform better when their learning skills are addressed properly. The normal farmers, rank very high regarding extraversion and openness. This opens the possibility that these farmers are not highly interested in improving starch potato cultivation but have their interests in other aspects of their farm or life. Learning styles and personality have impact on use of DSS's; especially anxiety is one of the scales, which blocks effectively the usage of DSS's. All these factors may be taken into account when redesigning and/or personalizing a DSS.

\subsection{Data COlLECtion}

According to Herder and Juvina (Herder \& Juvina, 2005), user's navigation behaviour can be modelled using syntactic information ("e.g. which links are followed, what does the navigation graph look like, what is the time that users spent on each page"), semantic information ("i.e. what is the meaning of the information that the user encountered during navigation"), and pragmatic information ("i.e. what is the user using the information for, what are the user's goals and tasks").

Because we want to obtain as much information as possible, we record data on two types of log files. Each session is "mapped" to these two types of files:

1. the movement file (MOV): it contains information concerning the movement from one 'source' page to a 'destination' page, such as time stamp, name of source page, name of destination page, and the button used (see Table III).

\footnotetext{
${ }^{1}$ Hierarchical cluster analysis based on Ward's method, k-means cluster analysis, and a two-step cluster analysis were used to recognise the different farmer clusters within the Dutch AVEBE farmer population.
} 
2. the variable file (VAR): it contains data information concerning actions done within pages, such as time stamp, page name, field/variable name and field value, role. This file may provide both syntactic and pragmatic information. However, in this paper we will not employ these information.

Table III. An example of a MOV log file.

\begin{tabular}{|l|l|l|l|}
\hline Time stamp & FromPage & ToPage & Button \\
\hline 2004-12-22 22:13:29 & field & pcn & NEXT_BUTTON \\
2004-12-22 22:13:35 & pcn & order & NEXT_BUTTON \\
2004-12-22 22:14:00 & order & yield & NEXT_BUTTON \\
2004-12-22 22:14:26 & yield & crop & NEXT_BUTTON \\
2004-12-22 22:16:16 & crop & yield & BACK_BUTTON \\
2004-12-22 22:16:25 & yield & crop & NEXT_BUTTON \\
2004-12-22 22:16:53 & crop & details (katinka_l) & CULTIVAR_LIST \\
2004-12-22 22:17:54 & details & allcultivars & DETAILS_TEXT \\
\hline
\end{tabular}

In Table III is shown an example of OPTIRas ${ }^{\mathrm{TM}}$ sequence of navigation, recorded in a MOV file. The user is accessing sequentially 7 types of pages, mostly by pressing the 'Next' button. The MOV file (Table III) is providing us with syntactic information, namely information needed to build navigation graphs (see Section 3).

Users can login in OPTIRas ${ }^{\mathrm{TM}}$ registering with their e-mails, but they can register also with an anonymous ID. The system provides this opportunity for the sake of user-friendliness, but also to protect farmers' privacy. The anonymous ID takes into account the IP number. This manner of anonymousiness provides advantages for the users, but bring disadvantages to the analysis. A user may login first time with his/her e-mail, the second time anonymously using computer $\mathrm{A}$, and the third time again anonymously using computer B; in the analysis, these three sessions will counts as sessions belonging to three separate users, and we cannot see anymore how a user eventually change his behaviour in time. Moreover, users can login by specifying their role, i.e. grower, scientist, extension worker, or other.

OPTIRas ${ }^{\mathrm{TM}}$ usage is expected to be used within two peak periods (i) November/December and February (period for purchasing crops, expected to be the peak period) and (ii) April and August/September (period when the yield is actually obtained). Farmers may compare the obtained yield with the advice given earlier by OPTIRas ${ }^{\mathrm{TM}}$. 
We are inspecting only the MOV log files starting with the 18th of December 2004 (the date when OPTIRas ${ }^{\mathrm{TM}}$ became on-line) and stopping with the 30th of May 2006, thus we have two peak periods: (i) December 2004 - February 2005 and (ii) November 2005 - February 2006. In total, 763 user sessions belonging to 501 individual users have been logged and employed in the analysis.

\section{Mining navigational behaviour with process mining techniques}

Different methods have been used to investigate the navigation patterns of users, by using logged data. Some researchers constructed aggregated measures (Herder, 2002), while others developed graphical and numerical methods for depicting navigational patterns (John E. McEneany, 2001). In our case, we use process mining techniques to inspect navigational patterns. Namely, we want to answer to the following questions:

1. What kind of navigation patterns do farmers show?

2. What kind of navigation patterns shows each target group (e.g. growers, extension workers, scientists)?

3. Knowing that farmers can be categorised in four groups (e.g. top farmers, quality, quantity and normal farmers), what kind of navigation patterns are associated with each of these groups?

Process mining techniques allow for extracting information from event logs. For example, the audit trails of a workflow management system or the transaction logs of an enterprise resource planning system can be used to discover models describing processes, organisations, and products. Moreover, it is possible to use process mining to monitor deviations (e.g., comparing the observed events with predefined models or business rules). For an overview of process/workflow mining issues see (Aalst et al., 2003). In this paper we use process mining to inspect navigational behaviour, which, to our knowledge, is a new application of this technique ${ }^{2}$. For this purpose we use the ProM framework (The ProM framework, n.d.).

The ProM pluggable framework offers a wide variety of process mining techniques and it is easy to add new plug-ins without the need to

\footnotetext{
2 Process mining can be also used for mining the organisational and case perspectives (Aalst \& Weijters, 2004b). In case of organisational perspective, the result of the mining process is comparable with a social network (Aalst \& Song, 2004).
} 
recode parts of the system. Moreover, it allows for the import from and the export to a wide variety of formats and systems (ranging from enterprize information systems and workflow products to social network software and classical mining tools) and provides advanced visualization and verification capabilities (The ProM framework, n.d.). ProM framework is equipped with a plethora of algorithms.

The choice of the most appropriate algorithm for a certain problem is not always straightforward. We use genetic process mining (Medeiros, 2006) and heuristic mining (Weijters \& Aalst, 2003). Genetic process mining algorithms are a search technique that mimics the process of evolution in biological systems. Genetic based algorithms for process mining developed by Alves de Medeiros and implemented in the ProM framework provide good heuristics that can tackle all relevant process-related structural constructs (i.e. sequences, parallelism, choices, loops). Moreover, these algorithms are equipped with analysis metrics that assess how complete, precise and folded the resulting process representations are (Medeiros, 2006). The major drawback of these algorithms is the high computational time; however, this is not a major problem for us, because the analysis is not supposed to be performed frequently. Rather, we are interested about the quality of the mined models, thus information such as completeness and preciseness are more than welcome. The second kind of process mining algorithm we use is the heuristic mining developed by Weijters (Weijters \& Aalst, 2003), also implemented in the ProM framework. We choose heuristic mining because for its robustness for noise and exceptions. Because the heuristic mining is based on the frequency of patterns it is possible to focus on the main behaviour in the event log.

\subsection{Mining the navigational Pattern of ALl USERS}

This section concentrates on the navigational patterns exhibited by all users. In Figure 4 is shown the result of applying the ProM genetic process mining plug-in, using the MOV log file. The focus is to grasp the patterns of navigation from one page to another (we use the FromPage field of the MOV file, see Table III). The file consists on 763 user sessions, belonging to 501 individual users. The rectangles refer to transitions or activities, in our case to page names, such as Field, Order, etc. There are two special activity names, ArtificialStartTask and ArtificialEndTask that refer to a generic start or end page. The term 'complete' refers to a finished action (in terms of a finitestate machine, an activity can be in the states 'new', 'sent', 'active', 'suspended', 'complete', etc.). The number inside the rectangle shows how many times a page has been invoked. The arcs (associated with a 
number) between rectangles represent the occurrence of two pages and how often it happens. For instance in Figure 4, Pcn page occurred 522 times after Field page.

Although the picture from Figure 4 looks quite complicated, let us interpret it. We can determine "the most common pattern" which consists of ArtificialStartTask $\rightarrow$ Field (755), Field $\rightarrow$ Pcn (522), Pcn $\rightarrow$ Order (493), Order $\rightarrow$ Yield (453), Yield $\rightarrow$ Crop (385), Yield $\rightarrow$ Crop (562), Crop $\rightarrow$ Yield (141), and Yield $\rightarrow$ ArtificialEndTask (146). The sequence Field $\rightarrow$ Pcn $\rightarrow$ Order $\rightarrow$ Yield $\rightarrow$ Crop is actually the "prescribed" order of pages in OPTIRas ${ }^{\mathrm{TM}}$, e.g. the order in which pages are presented in OPTIRas ${ }^{\mathrm{TM}}$.

We remark the reversed links between Crop $\rightarrow$ Yield(141), Yield $\rightarrow$ Order(177), and Yield $\rightarrow$ Pcn(146). The first two reversed links are not so "interesting"; however the third, Yield $\rightarrow$ Pcn provides an interesting insight, which may suggest that users are changing PCN values to observe the impact on calculating the yield (the higher the values of PCN, the smaller the yield). Noticeable is also the high frequent self-recurrent link to Yield(562).

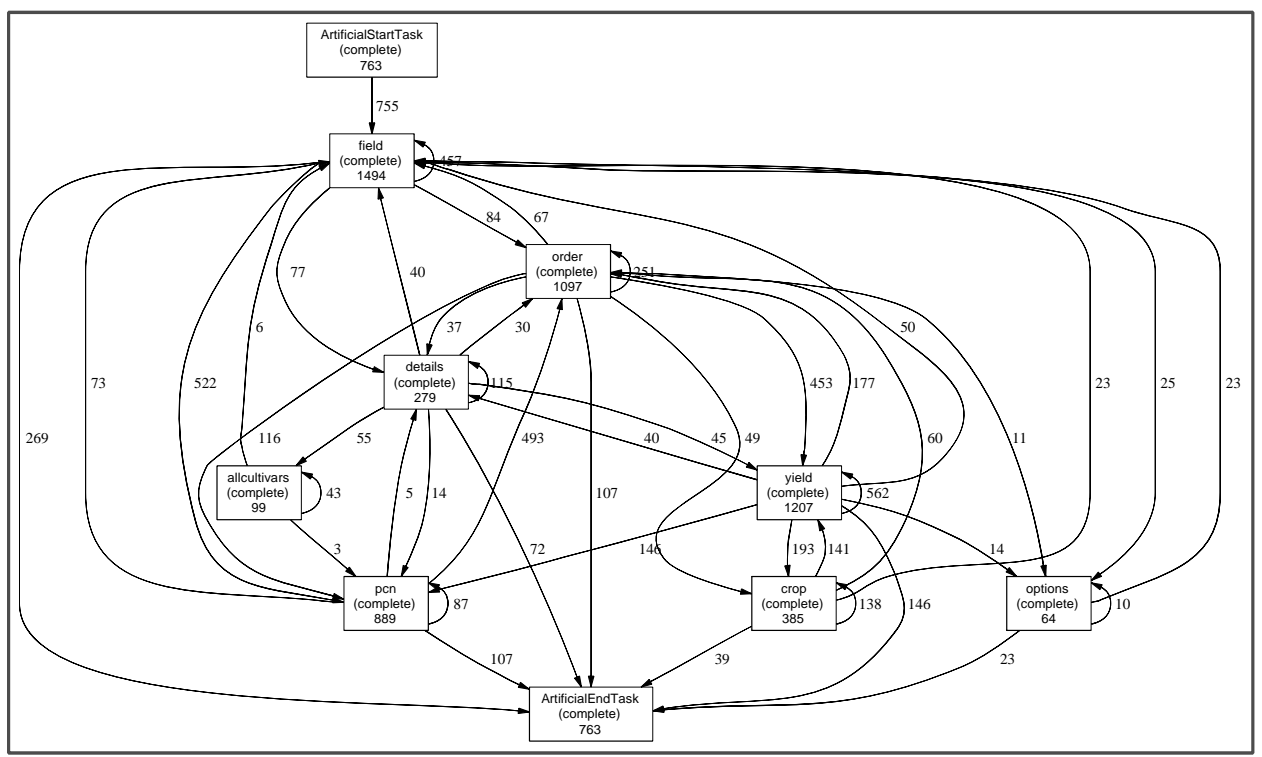

Figure 4. The mined behaviour of all farmers (run of the genetic algorithm).

The property of the genetic algorithm is that it expresses the most frequent behaviour (Medeiros, 2006). We want to compare these results with those of the heuristic miner, which is also focusing on the main behaviour in the event log, and is robust to exceptions. In Figure 5 we see a simpler picture of the entire user group behaviour. We observe 


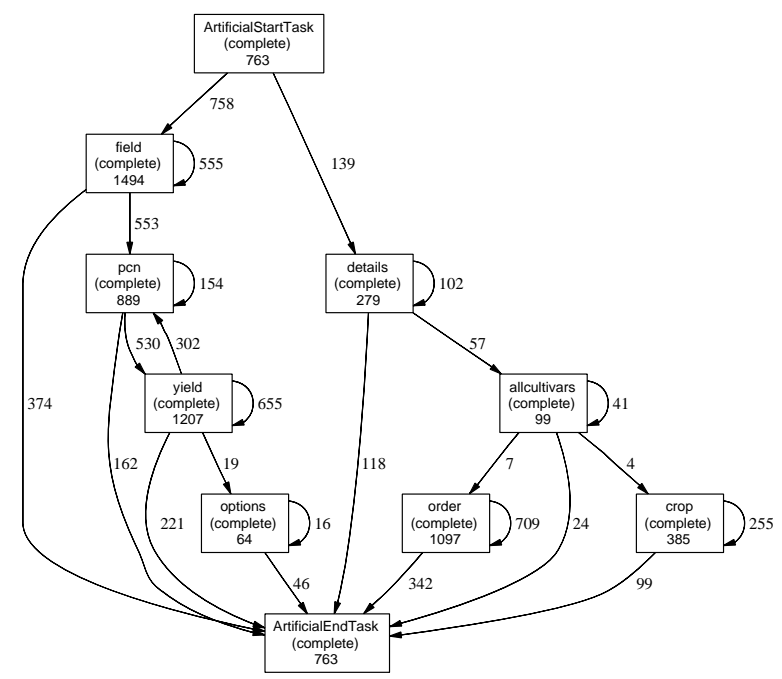

Figure 5. The mined behaviour of all farmers (run of the heuristic mining algorithm).

a comparable behaviour ${ }^{3}$ with that from Figure 4, but with some noticeable exceptions, such as the Order page, which is not in sequence anymore with Pcn and Yield pages, and the Details page, which seems to be an alternative to the prescribed path that starts with the Field page.

The interpretation of these findings is that farmers use in general the prescribed sequence of page invocation. The directed graphs from both Figure 4 and Figure 5 reveal that 'Action' pages are predominating: in Figure 4, the sum of incoming arcs from 'Action' pages to the end activity ArtificialEndTask is $269+107+107=483$, compared with 'Target' pages, the sum of incoming arcs from 'Target' pages to the end activity ArtificialEndTask is $146+39=185$. Similarly, in Figure 5, the sum of incoming arcs from 'Action' pages to the end activity ArtificialEndTask is $374+162+342=878$, compared with 'Target' pages, the sum of incoming arcs from 'Target' pages to the end activity ArtificialEndTask is $221+99=320$.

The first conclusion would then be that pages from category 'Target' are visualized significantly less than 'Action' pages is a disappointing result, given the fact that the main goal of OPTIRas ${ }^{\mathrm{TM}}$ is to provide detailed information about yield. Second, after each page a significant number of sessions stop (in Figure 5, 374 after page Field, 162 after

\footnotetext{
3 The two algorithms result into comparable outcomes; however, there are clear differences between the two models, because of the different mining algorithms used. For more information about these algorithms, see (The ProM framework, n.d.).
} 
page $P(n)$. However, we also see that whenever users do not 'give up' in early stages, and visualize 'Target' pages, they show a relevant activity. Namely, they recall repeatedly the Yield page, they revisit pages not directly linked (the reverse link Yield $\rightarrow$ Pcn), and also directly linked. This fact illustrates that whenever users reach a 'Target' pages, their interest may rise. The Details page is visited unexpectedly often: 279 times, and even forms a path containing only one page (e.g. the path ArtificialStartTask, details and ArtificialEndTask (see Figure 5). This suggests that users are interested in information about different cultivars.

\subsection{Mining the navigational Patterns of target Groups}

In this section we are answering to the next question mentioned in the beginning of Section 3, namely we are investigating the patterns of behaviour of different target groups. These target groups are growers, extension workers, and scientists. Whenever a user login into OPTIRas ${ }^{\mathrm{TM}}$, he/she has to specify his/her role, e.g. growers, extension worker, scientist or others. The distribution of sessions is as following: from all 763 sessions, 535 are sessions belonging to growers, 59 are sessions coming from extension workers and 72 are results of scientists usage. The rest of 97 sessions which are not labelled, are left them out from the analysis.

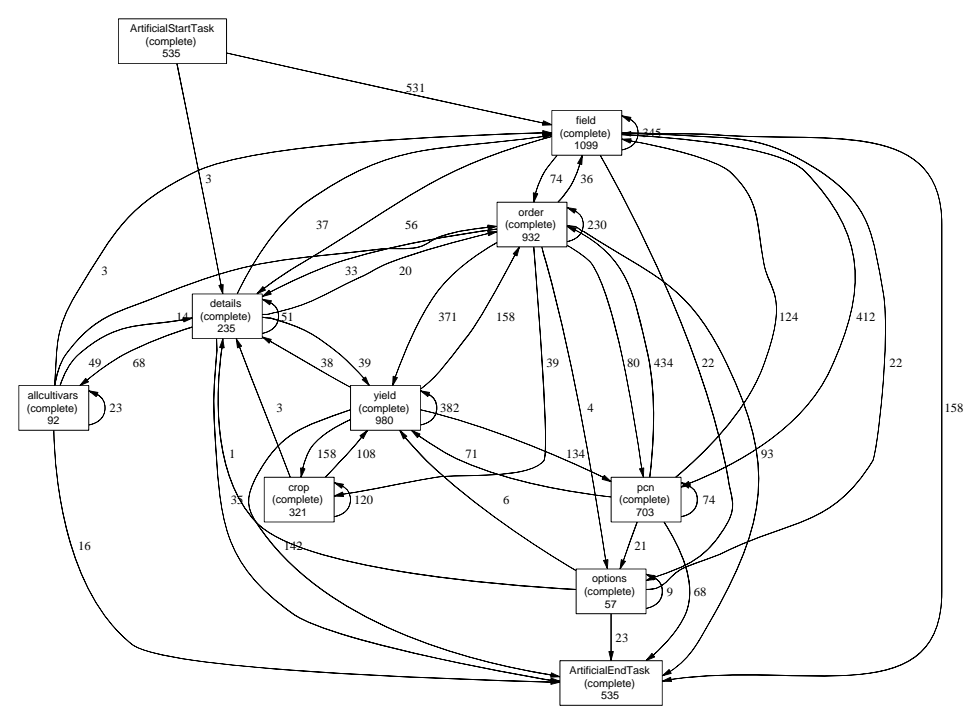

Figure 6. The mined behaviour of growers using the genetic algorithm. 


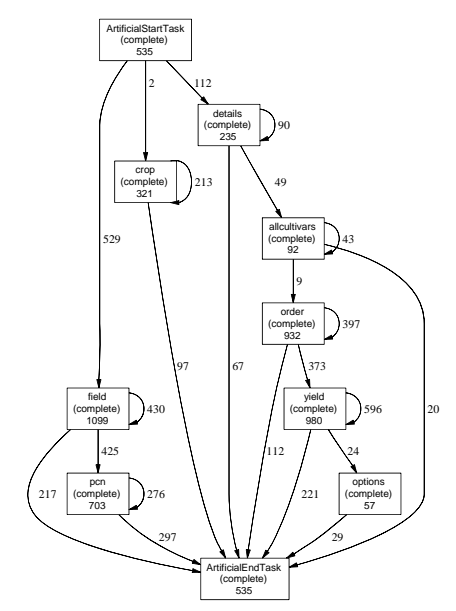

Figure \%. The mined behaviour of growers using the heuristic algorithm.

In Figure 6 is shown the navigation pattern of growers, run with the genetic algorithm. This pattern is comparable with that of all farmers from Figure 4, which is not surprising (growers form the majority of users). Also in case of growers, 'Action' pages are predominating (in Figure 6, the sum of incoming arcs from 'Action' pages to the end activity ArtificialEndTask is $158+68+93=319$ ), compared with 'Target' pages (the sum of incoming arcs from 'Target' pages to the end activity ArtificialEndTask is 142).

However, the run of the heuristic algorithm (see Figure 7) depicting the navigation pattern of growers is providing more insights into the most generic behavior: there seems to be differences between all farmers (Figure 5) and growers (Figure 7). For instance, in case of growers, the most used path is constituted by Field and Pcn pages, without the Yield page. This fact could be interpreted that the majority of growers are not reaching the Yield page, and thus we may doubt over fulfilling the goal of the DSS. Also, the navigational pattern of growers does not contain loops (see in Figure 5 the loop between Pcn and Yield), except from self-loops.

Inspecting further the navigation patterns of the extension workers, we see for instance from the run of the heuristic algorithm (see Figure 9) that the most common behavior is to stop after visiting the Field page. The other path starting with the Pcn page is less easy to be interpreted (without any incoming arc); however, we can note the loop between Pcn, Order and Yield, which illustrate a rather complex behavior.

The most generic behavior of scientists is the most complex (see Figure 11): there are two loops, e.g. (i) Field and Pcn and (ii) Or- 


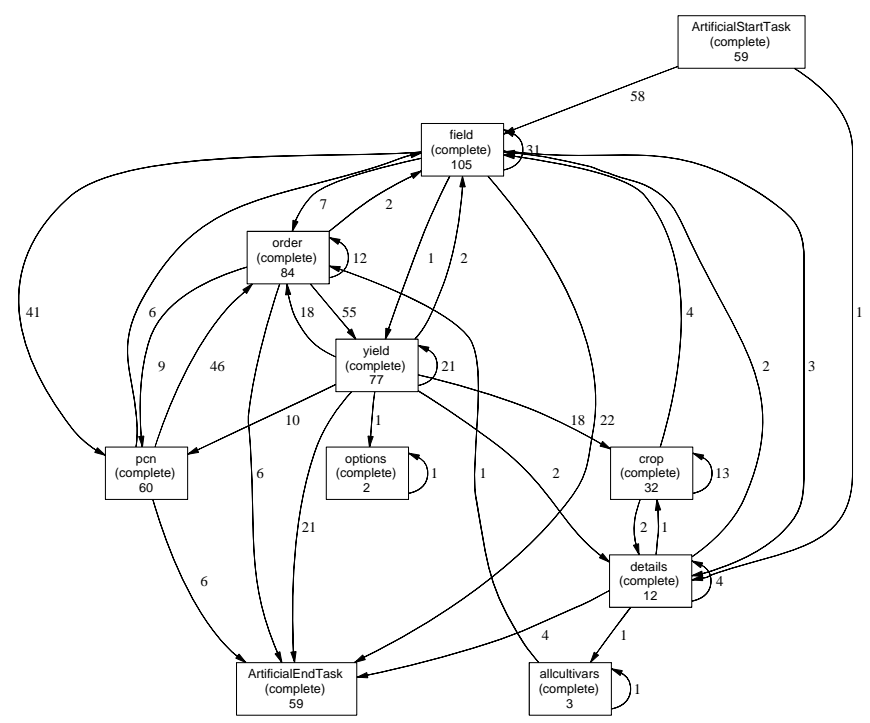

Figure 8. The mined behaviour of extension workers using the genetic algorithm.

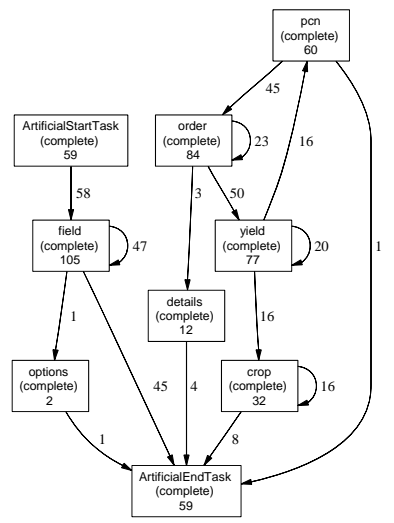

Figure 9. The mined behaviour of extension workers using the heuristic algorithm.

der and Yield. This could illustrate the fact that scientists, instead of only following the prescribed behavior, they are checking the effects of changing value parameters. Also, mostly of users belonging to this group are visiting 'Target' pages ( Yield and Crop), which may suggest that they easily understand the goal of this DSS.

\subsection{Mining the navigational Patterns of FARMer Groups}

In Section 2.3 we describe how farmers can be split in different groups, based on quantity and quality of the starch potato they produce. 


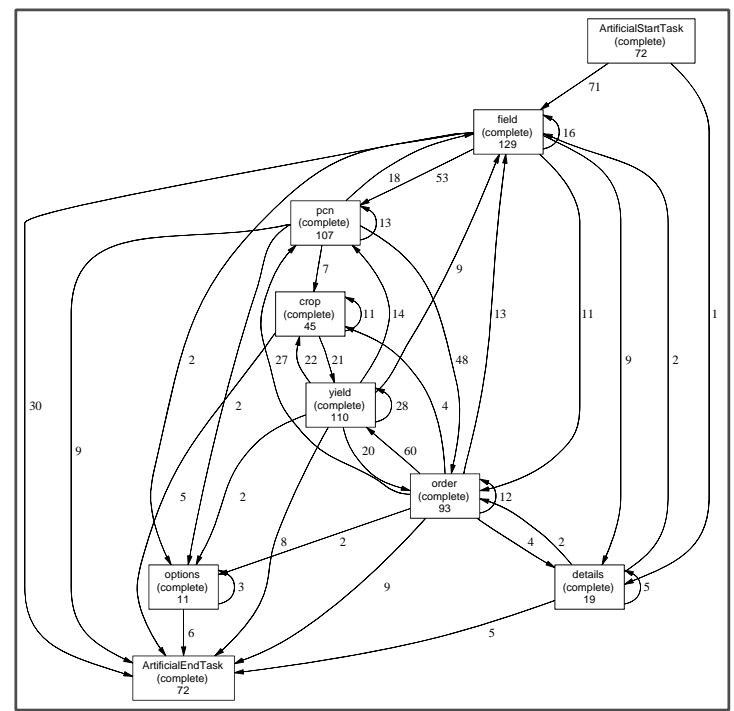

Figure 10. The mined behaviour of scientists, using the heuristic algorithm.

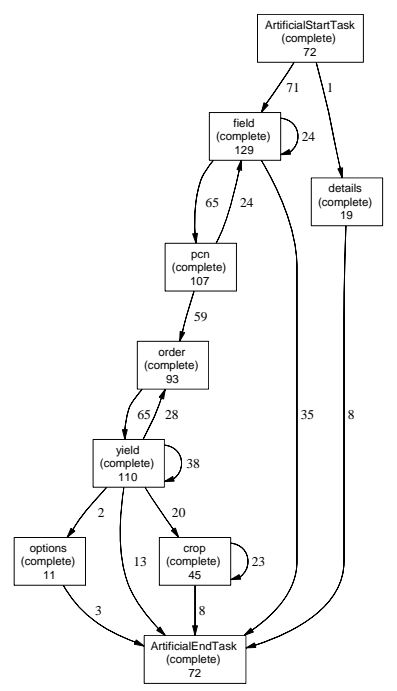

Figure 11. The mined behaviour of scientists, using the heuristic algorithm.

Namely, they can be categorized as "top-farmers", "quantity farmers", "quality farmers", and "normal farmers".

The third question we ask in the beginning of Section 3 is related to navigational patterns and these farmer groups. Connecting information from a research done earlier (N. Faber et al., 2006) and e-mail addresses used for logging in, we identified twenty-two sessions belonging to "top- 


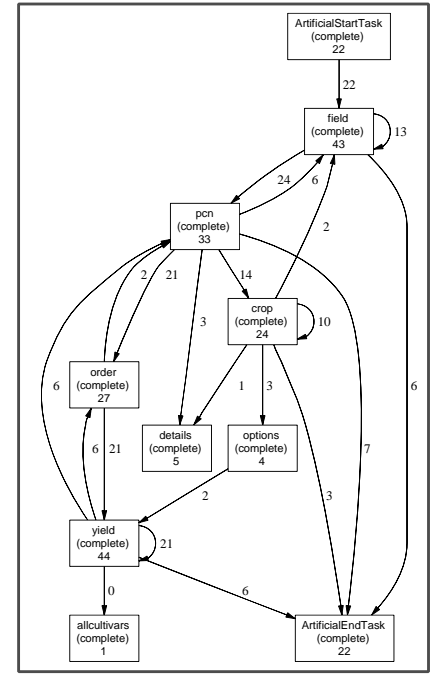

a. top farmers

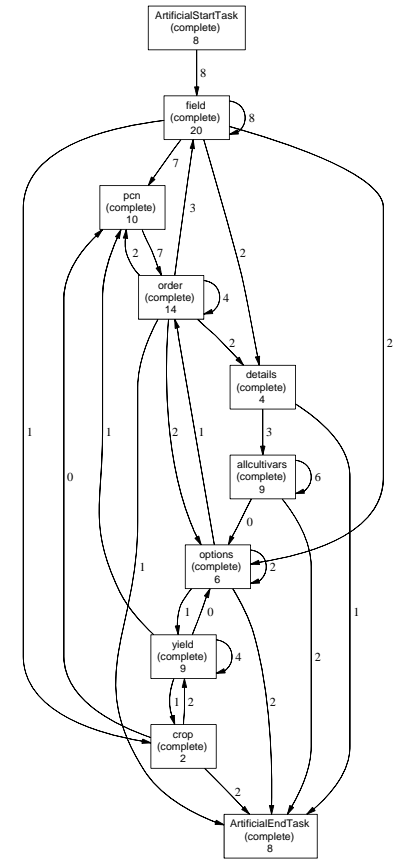

b. quality farmers

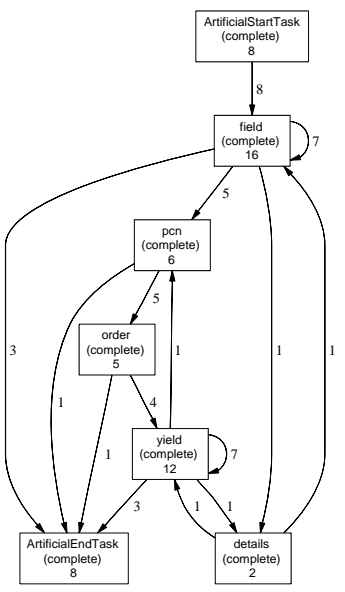

c. quantity farmers

Figure 12. The mined behavior for clusters 'top farmers', 'quality farmers' and 'quantity farmers', using genetic algorithm.

farmers", eight sessions to "quantity" farmers, and eight sessions for "quality farmers". Unfortunately, during the period logged, only one "normal" farmer logged in with his e-mail address, and we left out this session from the analysis. Because per each farmer group there is a small number of sessions, we do not aim to reveal the general navigation patterns of different farmer groups; rather we intend to illustrate a methodology of providing navigational patterns, that can be used for personalization purposes.

In Figure 12 are shown the navigational patterns for "top-farmers" (a.), "quality farmers" (b.), and "quantity-farmers" (c.). Looking closer to these three navigation patterns, we note that "top-farmers" exhibit a behavior comparable with scientists, characterized by visiting often 'Target' pages, and looping (e.g. (i) Field and Pcn and (ii) Order and Yield). "Quality farmers" show a "spread" behavior, without clear defined patterns. By contrary, "quantity farmers" seems to follow in majority the prescribed order of pages, e.g. Field, Pcn, Order, Yield. 


\section{Conclusions}

In this paper we provided more insights about patterns of navigation behavior, in case of a specific group of users, namely farmers. Using logs resulted from a decision support system called OPTIRas ${ }^{\mathrm{TM}}$, that aids farmers in their cultivar selection activities. We analysed these logs using process mining techniques.

With respect to all farmers, they use the prescribed order of pages, e.g. Field, Pcn, Order, Yield. We found out that often, farmers visualize just the first page, and then leave the system. With respect to goal fulfilling, the invocation of 'Action' pages predominates, while the invocation of 'Target' pages have a smaller proportion than expected. This corroborates with our assumption that users of OPTIRas ${ }^{\mathrm{TM}}$ spend much time in the Intelligence phase (Simon, 1977), exploring the domain of cultivar selection. This shows that users do not realize fast enough what the goal of OPTIRas ${ }^{\mathrm{TM}}$ is, and what kind of advantages they may have when using this system. This leads to a lack of interest, which as consequence means that users stop using the system. The fact that often the Details page is visited in the beginning of farmer's sessions suggests that in some cases, users are more interested in information about different cultivars, rather than cultivar selection based on Yield or Crop figures.

Analysing logs of different target groups, we found that the most common behavior of growers shows difficulties in fulfilling the goal of OPTIRas $^{\text {TM }}$, e.g. visiting 'Target' pages, while the other two target groups, extension workers and scientists seems to manage better. Given the fact that OPTIRas ${ }^{\mathrm{TM}}$ was especially developed to support growers, it is a clear sign that some redesign actions are needed.

Interesting patterns are revealed by analysing logs belonging to different farmer groups. "Top-farmers" show a behavior comparable with scientists, namely visiting often 'Target' pages and looping, while "quantity farmers" show to follow the prescribed behavior, given by the order of pages. The obtained patterns also can reveal interesting characteristics with respect to learning styles. For instance, top farmers show a more complex behavior than quantity farmers, which could be connected with specific learning styles. However, more logs are needed to advance generic patterns of behavior of different farmer groups.

The results of our analysis can be used to support the redesign of DSSs in order to address specific farmer's characteristics. First, we suggest that in the very first page a farmer encounters when using the system, the goal of OPTIRas ${ }^{\mathrm{TM}}$ should be very explicit and clearly stated, perhaps illustrated with a short example. Second, depending on the target group, hints for the following steps should be given: 
e.g. in case of growers, what path should be followed to get the yield overview. For this, the use of a sitemap is regarded to be appropriate. Third, depending on the type of farmer (top/quality/quantity/normal farmer), emphasize should be placed on, or hints should be given to those aspects that mostly connect with specific business rules that determine a farmer to be a top/quality/quantity/normal farmer. Lastly, OPTIRas $^{\mathrm{TM}}$ should much stronger focus on the Intelligence phase of decision-making, i.e. informing farmers about the various topics involved in cultivar selection, instead of presenting itself as an instrument to make optimal choices.

As further research we plan to investigate into depth the navigation behavior of different farmer groups, e.g. top-farmers, quality, quantity and normal farmers, and especially to connect navigation patterns with learning styles.

As a final remark, although we refer to a specific class of users, namely farmers, we think that the methodology presented in this paper can be used in case of any group of users that, based on some criterion, can be split in sub-groups.

\section{Acknowledgment}

We want to thank Rob van Haren from AVEBE, The Netherlands for providing us access to the data, and Iain Milne from Scottish Crop Research Institute for enabling the logging function of OPTIRas ${ }^{\mathrm{TM}}$.

\section{References}

Aalst, W. van der, Dongen, B. van, Herbst, J., Maruster, L., Schimm, G., \& Weijters, A. (2003). Workflow Mining: A Survey of Issues and Approaches. Data and Knowledge Engineering, 47(2), 237267.

Aalst, W. van der, \& Song, M. (2004). Mining Social Networks: Uncovering interaction patterns in business processes. In B. P. J. Desel \& M. Weske (Eds.), International conference on business process management (bpm 2004) (Vol. LNCS 3080, p. 244-260). Springer-Verlag Berling Heidelberg 2004.

Aalst, W. van der, \& Weijters, A. (2004a). Process mining (Vol. 53; W. van der Aalst \& A. Weijters, Eds.) (No. 3). Elsevier Science Publishers, Amsterdam.

Aalst, W. van der, \& Weijters, A. (2004b). Process mining: A research agenda. Computers in Industry, 53(3), 231-244. 
Allan Leck Jensen. (2001). Building a web-based information system for variety selection in field crops-objectives and results. Computers and Electronics in Agriculture, 32, 195-211.

AVEBE. (n.d.). Optiras - rassenkeuze adviessysteem voor zetmeelaardappelen. http://vti10.vertis.nl.

B. Balajinah and S.V. Raghavan. (2001). Intrusion detection through learning behavior model. Computer Communication, 24, 12021212.

Berry, D. (1994). Involving users in expert systems development. Expert systems, 11, 23-28.

Bo Öhlmér and Kent Olson and Berndt Brehmer. (1998). Understanding farmers' decision making process and improving managerial assistance. Agricultural Economics, 18, 273-290.

Bollini, L. (n.d.). Web interface design based on cognitive maps: Generative dynamics in information architecture. The 6th Generative Art Conference - GA2003.

Faber, N., Jorna, R., Haren, R. van, \& Maruster, L. (2006). Knowledge and knoweldge use for sustainable innovation: the case of starch potato production; achieving more with less. In Proceedings of the 11th international symposium on the management of industrial and corporate knowledge. Stellenbosch, Centre for Knowledge Dynamics and Decision-making.

Faber, N. R. (2006). Knowledge in sustainable behaviour. using knowledge-based decision support systems for the improvement of sustainability. Unpublished doctoral dissertation, University of Groningen.

Farm Information Systems, U. N. C. R. in. (2000). Farm information sytems: Their development and use in decision-making (Research Bulletin 601 No. Publication No. 345). Iowa State University, Ames, IS, USA.

H. Mintsberg and D. Raisingham and A. Thorêt. (1976). The structure of 'unstructured' decision process. Administrative Science Quarterly, 21, 246-275.

Hart, A., \& Wyatt, J. (1990). Evaluating black-boxes as medical decision aids: Issues arising from a study of neural networks. Medical Informatics, 15, 229-236.

Herder, E. (2002). Metrics for the adaptation of site structure. In Proc. of the German Workshop on Adaptivity and User Modeling in Interactive Systems ABIS'02, Hannover (p. 22-26).

Herder, E., \& Juvina, I. (2005). Discovery of individual user navigation styles. In Proc. of the Workshop on Individual Differences Adaptive Hypermedia, Eindhoven 2005. 
I. Thysen. (2000). Agriculture in the information society. Journal of Agricultural Engineering Resources, 76, 297-303.

John E. McEneany. (2001). Graphic and numerical methods to assess navigation in hypertext. International Journal of Humancomputer Studies, 55, 761-786.

Johnson, G., Halter, A., Jensen, H., \& Thomas, D. (1961). A study of managerial processes of midwestern farmers. Ames, Iowa: Iowa State Press.

Juvina, I., \& Herder, E. (2005a). The impact of link suggestion on user navigation and user perception. In Proceedings of user modelling 2005 (Vol. LNCS 3538, p. 483-492). Springer-Verlag.

Juvina, I., \& Herder, E. (2005b). Individual differences and behavioral aspects involved in in modeling web navigation. In Proccedings of user-centered interaction paradigms for universal access in the information society (Vol. LNCS 3196, p. 77-95). Springer-Verlag.

Juvina, I., \& Oostendorp, H. van. (2005). Bringing cognitive models into the domain of web accesibility. In Proceedings of HCII2005 conference, Las Vegas, USA.

Juvina, I., Oostendorp, H. van, Karbor, P., \& Pauw, B. (2005). Toward modeling contextual information in web navigation. In Proceedings of CogSci'05 Stresa, Italy.

Kerr, D. (2004). Factors influencing the Development and Adoption of Knowledge Based Decision Support Systems for Small, OwnerOperated Rural Business. Artificial Intelligence Review, 22, 127147.

Klein, M., \& Methlie, L. (1995). Knowledge-based decision support systems: with applications in business. Chichester: John Wiley \& Sons.

Kuhlmann, F., \& Brodersen, C. (2001). Information Technology and farm management: development and perspectives. Computers and Electronics in Agriculture, 30, 71-83.

Kun Chang Lee and Sangjae Lee. (2003). A cognitive map simulation approach to adjusting the design factors of the electronic commerce web sites. Expert Systems with Applications, 24, 1-11.

M. Rauterberg. (1993). AMME: an Automatic Mental Model Evaluation to analyse user behavior traced in a finite, discrete state space. Ergonomics, 36, 1369-1380.

M. Rauterberg and S. Schluep and M. Fjeld. (1998). Modeling of cognitive complexity with Petri nets: an action theoretica approach. Cybernetics and Systems, 2, 842-847.

Manfred Thüring and Jörg Hannemann and Jörg M. Haake. (1995). Hypermedia and Cognition: Designing for Comprehension. Communnication of the ACM, 38(8), 57-66. 
Medeiros, A. de. (2006). Genetic Process Mining. Unpublished doctoral dissertation, University of Eindhoven.

Menasalvas, E., Millan, S., Perez, M., Hochsztain, E., Robles, V., Marban, O., et al. (2003). Beyond user clicks: an algoritm and an agent-based arhitecture to discover user behavior. In Proceedings of ECML/PKDD, First European Web Mining Forum.

Myra Spiliopoulou and Carsten Pohle. (2001). Data Mining to Measure and Improve the Success of web Sites. Data Mining and Knowledge Discovery, 5(1-2), 85-114.

Neil A. Ernst and Margaret-Anne Story and Polly Allen. (2005). Cognitive support for ontology modeling. International Journal of Human-computer Studies, 62(5), 553-577.

Pauline A. Smith. (1996). Toward a practical measure of hypertext usability. Interacting with Computers, 8(4), 365-381.

Peter Hayman. (2003). Decision support systems in Australian dryland farming: A promising past, a disspointing present and uncertain future. http://www.cropscience.org.au/icsc2004/pdf/1778_haymanp.pdf.

Pieters, D. (2005). Stijlvol leren en kennis overdragen: een onderzoek naar leer- en persoonlijkheidsstijlen bij zetmeelaardappeltelers van avebe. Master thesis, University of Groningen, Groningen, The Netherlands.

The ProM framework. (n.d.). http://www.processmining.org.

Rauterberg, G. (2000). How to characterize a research line for user-system interaction (IPO Progress Report No. 35). IPO University of Eindhoven.

S. Fountas and D. Wulfsohn and B.S. Blackmore and H.L. Jacobsen and S.M. Pederson. (2006). A model of decision-making and informations flows for infomation-intensive agriculture. Agricultural Systems, 87, 192-210.

Simon, H. (1977). The new science of management decision (revised ed.). Englewood Cliffs, New Jersey: Prentice-Hall, Inc.

Turban, E., \& Aronson, J. (2001). Decision support systems and intelligent systems. Upper Saddle River, N.J.: Prentice-Hall.

Urquhart, C., \& Rowley, M. (1999). Issues of Adoption of Information Technology (IT): Barriers and Rewards in the Sunshine Coast Sub Tropical Fruit Growers IT Group. .

Weijters, A., \& Aalst, W. (2003). Rediscovering workflow models from event-based data using Little Thumb. Integrated Computer-Aided Engineering, 10, 151-162. 
"Niels UMUAI paper".tex; 19/02/2007; 17:03; p.26 